\title{
Successful blunt recanalization of an obliterated long esophageal stricture by endoscopic rendez-
} vous

A 57-year-old man with a history of squamous cell carcinoma of the tongue treated with chemoradiation therapy presented to our group for management of a proximal esophageal stricture. Despite serial endoscopic dilations with submucosal steroid at outside facilities, the stricture progressed, ultimately obliterating luminal patency. Consequently, caloric intake was maintained through a percutaneous gastrostomy tube; however, the patient's intolerance of secretions led to frequent aspiration ( $\bullet$ Fig. 1 )

An antegrade-retrograde rendezvous technique of endoscopic therapy, first described by Van Twisk, was used to address the complex esophageal stricture [1]. A standard 9.8-mm gastroscope was passed per os in the antegrade position. A second endoscopist approached the esophageal stricture in a retrograde fashion with a slim 5.9-mm gastroscope through a wellhealed 20-F gastrostomy site. Transillumination and fluoroscopy were employed to assist with dissection trajectory and en face positioning of the antegrade and retrograde endoscopes prior to and throughout stricture manipulation (๑ Fig.2). A straight endoscopic retrograde cholangiopancreatography (ERCP) cannula was then positioned through the antegrade endoscope and blunt dissection of the stricture carried out while the retrograde endoscope visualized the resultant

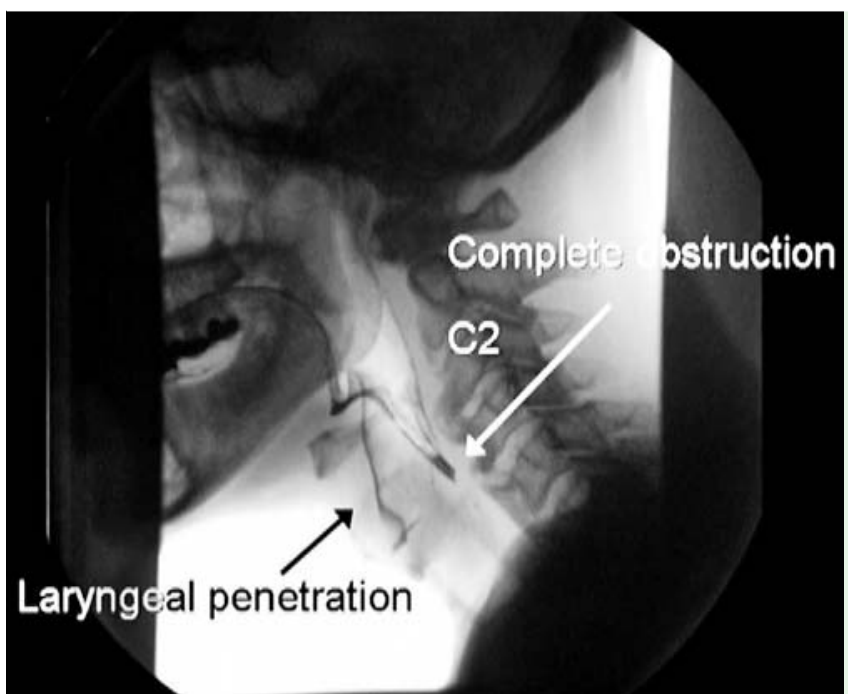

Fig. 1 Fluoroscopic image of the esophagus in a 57-year-old man with a history of chemoradiation for lingual squamous cell carcinoma demonstrating complete obstruction of contrast at the level of $\mathrm{C2} / \mathrm{C} 3$ and associated laryngeal penetration. tissue convexity and guided appropriate trajectory ( $\bullet$ Fig. 3 a and $\bullet$ Video 1 ). With the needle sheathed, a 19-gauge endoscopic ultrasound (EUS) needle was then used to complete the blunt dissection ( Fig.3b). Only after visualizing the needle sheath from a retrograde perspective was the needle passed to stabilize the antegrade position ( $\bullet$ Fig.3c). Through this needle a long 0.035 -inch hydrophilic guide wire was passed into the distal esophagus and stomach to allow for subsequent 8-mm controlled radial expansion balloon dilation and deployment of a nasogastric tube for maintenance of patency during subsequent serial endoscopies ( $\bullet$ Fig.4). The patient was prophylactically admitted for overnight observation and discharged the next day without complication. Serial over-the-guide wire dilations to $15 \mathrm{~mm}$ were carried out without issue. During each procedure contrast was infused to demonstrated patency without extravasation. With the assistance of speech/language physicians the patient now tolerates oral nutrition.

We have presented an uncomplicated recanalization of an obliterated long esophageal stricture by blunt dissection assisted by endoscopic rendezvous. Sharp dissection was avoided given the increased risk of inadvertent needle passage into the mediastinum with injury to surrounding vital structures. While complex, such a technique represents a reasonably safe therapeutic option with great potential for quality of life improvement.

Esophageal strictures develop in up to $4 \%$ of individuals receiving head and neck radiation of at least $4500 \mathrm{cGy}$ and upwards of $20 \%$ in those with concomitant use of chemotherapy [2]. While complete obliteration is rare, it is an appreciated entity
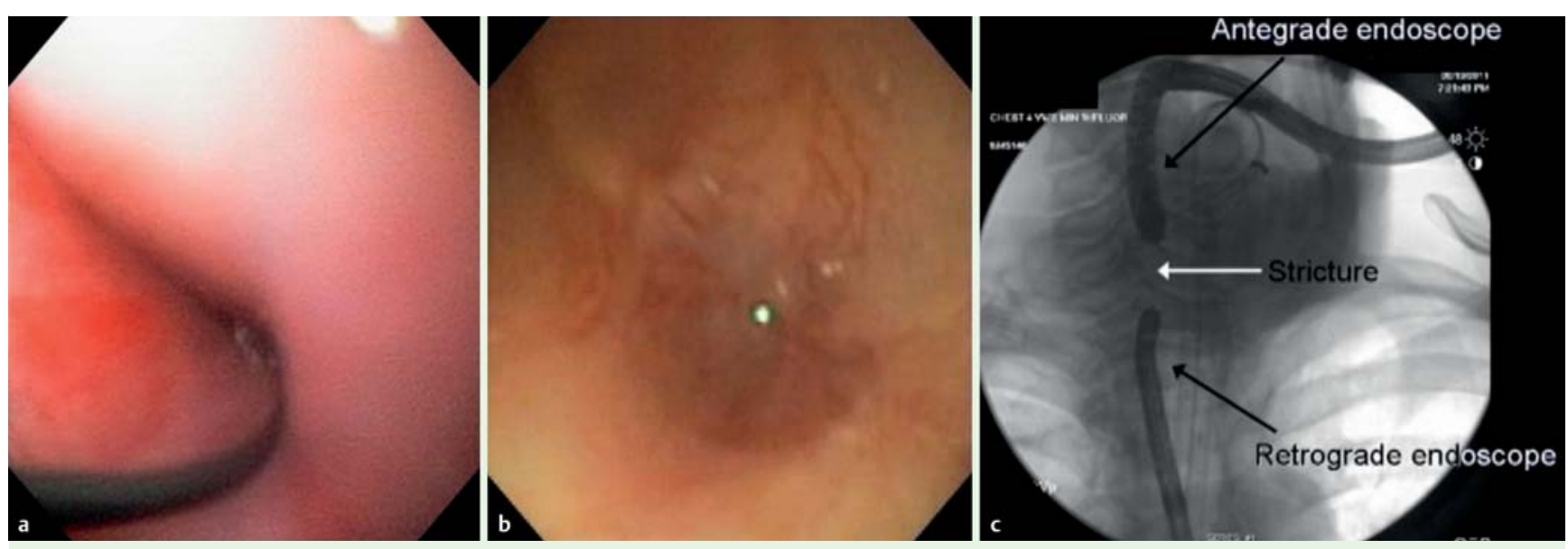

Fig. 2 Endoscopic views of the obliterated esophageal lumen: a antegrade position with soft wire probe; and $\mathbf{b}$ retrograde position. $\mathbf{c}$ Fluoroscopic image depicting the length (approximately $2.5 \mathrm{~cm}$ ) of the long esophageal stricture intervening between the antegrade and retrograde endoscopes. 

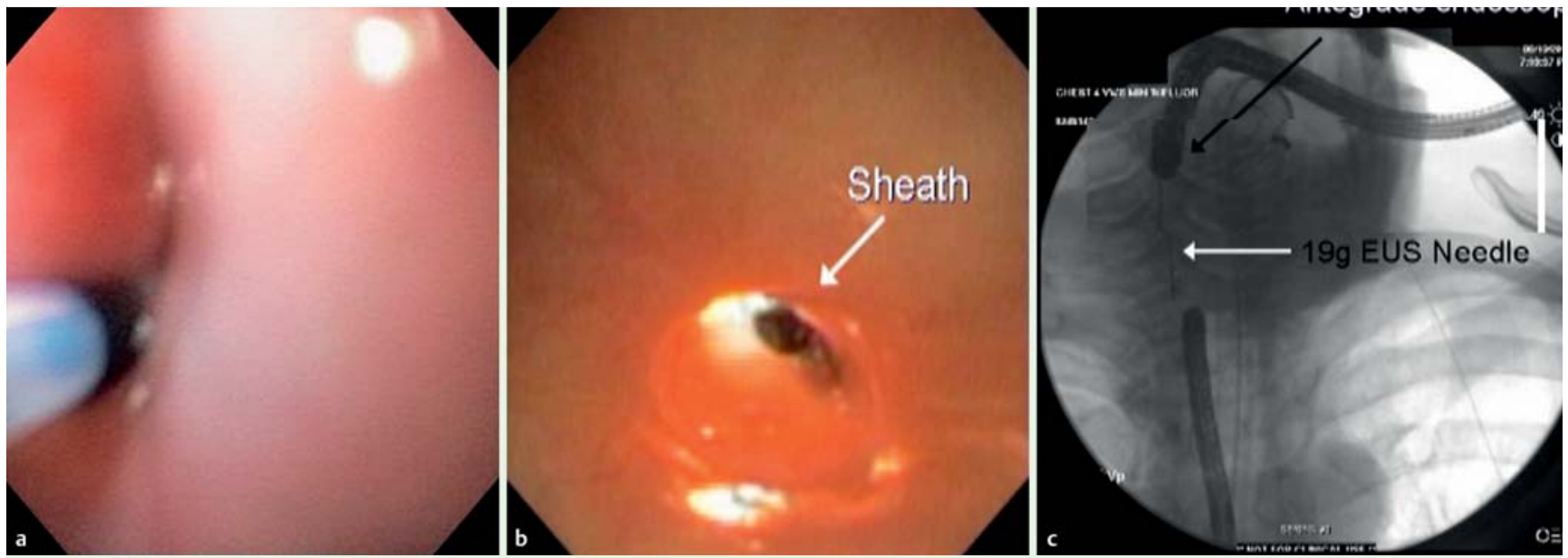

Fig. 3 a Blunt dissection of the stricture from the antegrade position utilizing a straight endoscopic retrograde cholangiopancreatography (ERCP) catheter. b Penetration of an endoscopic ultrasound (EUS) sheath from the retrograde position. c Fluoroscopic demonstration of needle passage from the antegrade to retrograde positions through the long obliterated lumen.
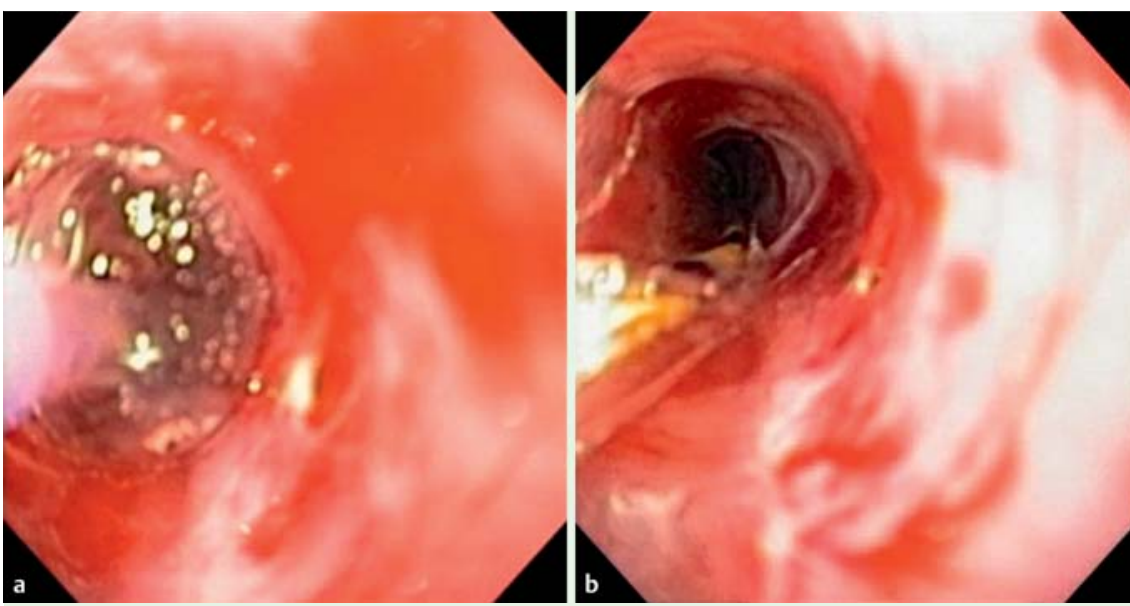

Fig. 4 a Through the scope controlled radial expansion balloon dilation of the esophageal stricture to $8 \mathrm{~mm}$. b Patent esophageal lumen immediately following initial balloon dilation.

with few traditional therapeutic options. Esophagectomy is complicated as the residual surrounding tissue is not ideal, and as the lumen is not patent, neither wire nor endoscopically guided dilations are possible. Limited literature exists regarding esophageal recanalization, in particular involving blunt dissection of a relatively long stricture such as demonstrated here [3-8]. In general complications appear limited, though subacute to long-term efficacy as measured by an ability to discontinue tube feeds is variable in part due to the lack of standardization.

Endoscopy_UCTN_Code_TTT_1AO_2AH

\section{Video 1}

Presentation of blunt recanalization of obliterated long esophageal stricture with schematic demonstration.

\section{Competing interests: None}

\section{S. K. Amateau', M. A. Khashab ${ }^{2}$}

${ }^{1}$ Department of Internal Medicine, Division of Gastroenterology and Hepatology, University of Colorado, Aurora, Colorado, USA

2 Department of Internal Medicine, Division of Gastroenterology and Hepatology, Johns Hopkins Hospital, Baltimore, Maryland, USA

\section{References}

1 van Twisk JJ, Brummer RJ, Manni JJ. Retrograde approach to pharyngo-esophageal obstruction. Gastrointest Endosc 1998; 48: 296-299

2 Coia LR, Myerson RJ, TepperJE. Late effects of radiation therapy on the gastrointestinal tract. Int J Radiat Oncol Biol Phys 1995; 31: $1213-1236$

3 Bueno R, Swanson SJ, Jaklitsch MT et al. Combined antegrade and retrograde dilation: a new endoscopic technique in the management of complex esophageal obstruction. Gastrointest Endosc 2001; 54: 368-372

4 Petro M, Wein RO, Minocha A. Treatment of a radiation-induced esophageal web with retrograde esophagoscopy and puncture. Am J Otolaryngol 2005; 26: 353-355

5 Maple JT, Petersen BT, Baron TH et al. Endoscopic management of radiation-induced complete upper esophageal obstruction with an antegrade-retrograde rendezvous technique. Gastrointest Endosc 2006; 64: 822-828

6 Moyer MT, Stack BCJr, Mathew A. Successful recovery of esophageal patency in 2 patients with complete obstruction by using combined antegrade retrograde dilation procedure, needle knife, and EUS needle. Gastrointest Endosc 2006; 64: 789-792

7 Al-Haddad M, Pungpapong S, Wallace MB et al. Antegrade and retrograde endoscopic approach in the establishment of a neo-esophagus: a novel technique. Gastrointest Endosc 2007; 65: 290-294

8 Schembre D, Dever JB, Glenn M et al. Esophageal reconstitution by simultaneous antegrade/retrograde endoscopy: re-establishing patency of the completely obstructed esophagus. Endoscopy 2011; 43: 434-437

\section{Bibliography}

DOI http://dx.doi.org/

10.1055/s-0032-1325967

Endoscopy 2013; 45: E49-E50

(c) Georg Thieme Verlag KG

Stuttgart · New York

ISSN 0013-726X

\section{Corresponding author}

\section{A. Khashab}

Johns Hopkins Hospital

1830 East Monument Street, Room 426

Baltimore, MD 21205

USA

Fax: +1-410-5020198

mkhasha1@jhmi.edu 